\title{
Resonance spectrum of the strongly interacting symmetry-breaking sector
}

\author{
J. R. Peláez* \\ Theoretical Physics Group, Lawrence Berkeley Laboratory, University of California, Berkeley, California 94720
}

(Received 23 September 1996)

\begin{abstract}
Within the chiral Lagrangian formalism it is possible to describe the general strongly coupled symmetrybreaking sector in terms of a few parameters. Based on a dispersive approach we study the resonance spectrum up to $3 \mathrm{TeV}$ in chiral parameter space. This procedure could also be useful to extract higher energy resonant behavior from low-energy collider data. It is also shown how the method reproduces the correct pole structure of resonances as well as other analytic features. The results also hint at a possible excluded region of parameter space. [S0556-2821(97)02307-2]
\end{abstract}

PACS number(s): 12.60.Cn, 11.15.Tk, 11.30.Qc, 12.39.Fe

\section{INTRODUCTION}

The main purpose of the next generation of colliders is to unveil the nature of the electroweak symmetry-breaking sector (EWSBS). Despite the remarkable success of the standard model (SM) with the present precision electroweak data, the mechanism responsible of this breaking remains unknown. There are, however, many theoretical models which, very roughly, can be divided in two categories: weakly or strongly coupled.

In the weak case light particles are expected below the $\mathrm{TeV}$ scale. Typical examples are the minimal SM (MSM) with a light Higgs boson or most supersymmetric models. These models have become very popular and have been studied in great detail. That is not possible in the strong case, where the strength of the interactions makes the usual perturbative approach unreliable. In particular, there are no light particles to control the generic enhancement of gauge boson production. As a consequence, the perturbative calculations suffer from severe unitarity violations. Nevertheless, such an enhancement would be the experimental signature of a strong EWSBS. The most promising process is longitudinal gauge boson scattering, where the most striking feature would be the appearance of heavy resonances.

There are also several models of strongly coupled EWSBS's, like the MSM with a heavy Higgs boson, technicolor, composite models, etc. From very general symmetry considerations all of them share the same dynamics at low energies [1]. However, the predictions of these models can vary greatly from one another.

Several years ago it was introduced a theoretical framework that is able to describe generically the strong interactions of electroweak gauge bosons [2]. It is based on chiral perturbation theory (ChPT) [3], which works remarkably well for pion physics. The idea is to write an effective chiral Lagrangian including operators up to dimension four [4]. The form of the terms is only constrained by symmetry considerations which are common to any strong EWSBS. Thus, using this Lagrangian it is possible to mimic the low-energy

\footnotetext{
*On leave of absence from Departamento de Física Teórica, Universidad Complutense, 28040 Madrid, Spain. Electronic address: pelaez@theorm.lbl.gov, pelaez@vxcern.cern.ch
}

behavior of any strong EWSBS. The difference between underlying theories appears through the values of the parameters in the chiral Lagrangian. There are already published chiral parameter estimates for several models such as the MSM with a heavy Higgs boson [5,6] or technicolor [7]. There are also studies which indicate that at least part of the interesting parameter space will be accessible at the CERN Large Hadron Collider (LHC) [8].

However, the usual chiral Lagrangian approach does not respect unitarity. At low energies the violations are very small, but they increase with the energy. As a consequence it is not possible to reproduce resonances unless it is modified. There are several ways to unitarize chiral amplitudes. Many of them are simple mathematical tricks whose results very frequently differ, which is an obvious criticism to such procedures. Nevertheless, over the last few years, a technique has been developed, known as the inverse amplitude method (IAM) [9-12], whose results have been successfully tested in ChPT. It is based on dispersion theory and it can accommodate all the analytic structure required to reproduce resonances. Namely, the elastic cut and poles in the second Riemann sheet. When applied to low-energy hadron physics, it is indeed able to reproduce the lightest resonances. The IAM seems very reliable at least at the qualitative level.

Concerning the EWSBS, the method was first applied to mimic a heavy Higgs boson and a QCD-like scenario at supercolliders [13]. The results of the IAM are once again consistent with the expected resonances.

The aim of this work is to explore the interesting part of the chiral parameter space using the IAM method. In so doing, we expect to obtain a description of the low resonance spectrum of the general strong EWSBS.

The paper is organized as follows. In Sec. II we discuss the theoretical framework used in this work. First we introduce the chiral formalism; next, we address some technical issues related to the equivalence theorem. We then define partial waves and state the unitarity problem. As a solution, the IAM is briefly reviewed, whose derivation is given in the Appendix. Section III is devoted to the IAM results. First, for reference models, we illustrate different analytical and physical features, such as saturation. We then show where these phenomena appear in parameter space. The problem of unitarity in the $I=2$ channel and whether it can be used to exclude part of the parameter space is also addressed in Sec. 
III. In Sec. IV we discuss these results and we gather them in the conclusion.

\section{RESONANCES IN THE CHIRAL FORMALISM}

\section{A. The chiral Lagrangian}

Let us remember that we have to break the $\mathrm{SU}(2)_{L} \times \mathrm{U}(1)_{Y}$ gauge symmetry down to $\mathrm{U}(1)_{\mathrm{EM}}$. Therefore we need a global breaking from a group $G$ down to another $H$. It should provide three Goldstone bosons (GB's) that will become the logitudinal components of the gauge bosons through the Higgs mechanism. We also want to include the custodial $\mathrm{SU}(2)_{L+R}$, which naturally yields a $\rho \simeq 1$ parameter [14]. It can be shown that these constraints lead to $G=\mathrm{SU}(2)_{L} \times \mathrm{SU}(2)_{R}$ and $H=\mathrm{SU}(2)_{L+R} \quad[1,15]$. Thus, the GB fields $\pi^{i}$ can be seen as coordinates in the $G / H \sim \mathrm{SU}(2)_{L-R}$ coset. Hence, we will parametrize them in an $\operatorname{SU}(2)$ matrix as $U=\exp \left(i \pi^{i} \sigma^{i} / v\right)$. The parameter $v \simeq 256 \mathrm{GeV}$ plays here the same role as $f_{\pi}$ in ChPT and sets the scale of the EWSBS.

Within the chiral approach we build the low-energy Lagrangian as an expansion in derivatives (momenta) of the GB fields. Since we will work up to $O\left(p^{4}\right)$, we should look for a complete set of $\mathrm{SU}(2)_{L} \times \mathrm{U}(1)_{Y}$, Lorentz, $C$ - and $P$-invariant operators containing up to four derivatives. These have been obtained in [4], but they are too general for our purposes. Indeed, we want an exact $\mathrm{SU}(2)_{L+R}$ symmetry on the hidden sector once the gauge couplings $g$ and $g^{\prime}$ are set to zero. In addition, we are only interested in gauge boson elastic scattering and we can neglect $C P$-violating effects.

With those assumptions, the only operator that we can build with two derivatives is

$$
\mathcal{L}^{(2)}=\frac{v^{2}}{4} \operatorname{tr} D_{\mu} U D^{\mu} U^{\dagger},
$$

where $D_{\mu} U=\partial_{\mu} U-W_{\mu} U+U B_{\mu}$ is a covariant derivative with $W_{\mu}=-i g \sigma^{a} W_{\mu}^{a} / 2$ and $B_{\mu}=-i g \sigma^{3} B_{\mu} / 2$. It is important to observe that this Lagrangian only depends on the symmetry-breaking pattern and the scale. In this sense, the amplitudes obtained from $\mathcal{L}^{(2)}$ are universal. That is why they are called low-energy theorems (LET's) [1].

Notice also that the Lagrangian in Eq. (1) is that of the nonlinear $\sigma$ model and thus it is not renormalizable. In fact it is not possible to absorb the loop divergencies by introducing a finite set of new counterterms and constants. Nevertheless, we are only interested in the low-energy behavior and therefore it is enough to work up to a given order in the external momenta. For instance, if we want to obtain gauge boson scattering amplitudes at $O\left(p^{2}\right)$, the only contributions come from $\mathcal{L}^{(2)}$ at tree level. If we calculate at $O\left(p^{4}\right)$, we will have to consider the $\mathcal{L}^{(4)}$ Lagrangian at the tree level as well as $\mathcal{L}^{(2)}$ to one loop. These last contributions are divergent, but their divergencies can be absorbed in the $\mathcal{L}^{(4)}$ parameters. In this sense, the calculations are renormalizable and finite. This procedure can be generalized to $O\left(p^{N}\right)$, but we will work only up to $O\left(p^{4}\right)$.

There are many possible terms in the $\mathcal{L}^{(4)}$ Lagrangian [4]. However, according to the above restrictions, we are only interested in
TABLE I. Chiral parameters for different reference models.

\begin{tabular}{lcc}
\hline \hline & $L_{1}$ & $L_{2}$ \\
\hline MSM $\left(M_{H} \sim 1 \mathrm{TeV}\right)$ & 0.007 & -0.002 \\
QCD-like & -0.001 & 0.001 \\
\hline \hline
\end{tabular}

$$
\begin{aligned}
\mathcal{L}^{(4)}= & L_{1}\left(\operatorname{tr} D_{\mu} U D^{\mu} U^{\dagger}\right)^{2}+L_{2}\left(\operatorname{tr} D_{\mu} U D^{\nu} U^{\dagger}\right)^{2}+\operatorname{tr}\left[\left(L_{9 L} W^{\mu \nu}\right.\right. \\
& \left.\left.+L_{9 R} B^{\mu \nu}\right) D_{\mu} U D_{\nu} U^{\dagger}\right]+L_{10} \operatorname{tr} U^{\dagger} B^{\mu \nu} U W_{\mu \nu},
\end{aligned}
$$

where $W^{\mu \nu}$ and $B^{\mu \nu}$ are the strength tensors of the gauge fields.

Finally, let us remark that using these Lagrangians we will obtain the chiral amplitudes as truncated series in $s$, the usual Mandelstam variable. That is

$$
t(s) \simeq t^{(0)}(s)+t^{(1)}(s)+O\left(s^{3}\right),
$$

where $t^{(0)}(s)$ is $O(s)$ and reproduces the LET's. It is obtained from $\mathcal{L}^{(2)}$ at the tree level. The $t^{(1)}(s)$ contribution is $O\left(s^{2}\right)$ and comes from the $\mathcal{L}^{(4)}$ at the tree level and $\mathcal{L}^{(2)}$ at one loop. The loops yield logarithmic contributions which are very relevant at low energies. However, at higher energies our amplitudes behave essentially as polynomials in $s$.

\section{B. Chiral parameters}

In contrast with the $\mathcal{L}^{(2)}$ Lagrangian, the one in Eq. (2) is not completely fixed by symmetry and the scale. Indeed each operator has a parameter which depends on the specific breaking mechanism. Thus, for every strong EWSBS without relevant light modes and our assumed symmetry-breaking pattern, there should be a different set of chiral parameters. Notice, however, that nothing ensures the reciprocal. It is not clear that for every set of chiral parameters there should be an underlying consistent and renormalizable quantum field theory (QFT).

Unfortunately, the very nature of strongly coupled theories does not allow a calculation of these parameters. There are, however, estimates for the heavy Higgs MSM, which are obtained from a matching of one loop Green functions [5]. For the QCD-like model, they are obtained by rescaling the QCD parameters [12]. We will use these models as a reference and thus we have listed their parameters in Table I.

Very recently several studies have appeared concerning the LHC capabilities to determine these parameters in case there is a strong EWSBS [8]. Notice that their expected values are in the $10^{-2}$ to $10^{-3}$ range. Note also that the sign of the parameters may play an essential role. From these preliminary studies it seems that LHC could be able to reach $5 \times 10^{-3}$, even in the hardest nonresonant case. However, that will require two detectors taking data for several years at full design luminosity and the highest center-of-mass energy.

\section{The equivalence theorem}

As we have already seen, the most relevant modes of the EWSBS at low energy are the GB. However, once we include the electroweak interactions, the GB disappear from the physical spectrum and become the longitudinal components of the gauge bosons $\left(V_{L}\right)$. Somehow we can identify 
the GB and their behavior with that of the gauge bosons. The precise formulation of the previous statement is known as the equivalence theorem (ET) [16,17]:

$$
T\left(V_{L}^{a_{1}}, \ldots, V_{L}^{a_{n}}\right) \simeq\left(\prod_{j=1}^{l} K_{\alpha_{j}}^{a_{j}}\right) T\left(\pi_{\alpha_{1}} \cdots \pi_{\alpha_{n}}\right)+O\left(\frac{m}{E}\right),
$$

where $m$ is the mass of the gauge boson. The $K$ factors, which include renormalization and higher order $g$ effects, are basically $1+O\left(g^{2}\right)$ [18]. In short, the ET allows us to identify, at energies $E \gg m$, the longitudinal gauge boson amplitudes with those of their associated GB. It is very useful in two senses. First it allows us to link the physical measurements with the hidden sector. Second, it helps in the calculation of the $V_{L}$ amplitudes, which are much easier to obtain using scalar particles such as GB.

It is important to notice that the ET is a high-energy limit. In contrast, the chiral formalism is a low-energy approach. Nevertheless, it has been recently shown that there is a window of applicability for the ET together with the chiral approach $[15,19]$. The above equation remains valid, but only at lowest order in $g$ and $g^{\prime}$.

In the following sections we will be thoroughly using the ET. Therefore we will work at lowest order in the electroweak couplings. As a consequence, only $L_{1}$ and $L_{2}$ will be relevant for our calculations.

\section{Partial waves, unitarity, and resonances}

As long as we have an $\mathrm{SU}(2)_{L+R}$ symmetry in the EWSBS, we can also define a weak isospin $I$. In analogy to $\pi \pi$ scattering, we have three possible weak isospin channels $I=0,1,2$. It is then usual to project the amplitudes in partial waves with definite angular momentum $J$ and isospin $I$. At low energies we are only interested in the lowest $J$, and thus we will study the $t_{I J}=t_{00}, t_{11}$, and $t_{20}$ partial waves. Their expressions for the EWSBS are given in [13]. Customarily the results of elastic scattering are presented in terms of their complex phases, which are known as phase shifts.

As we have already remarked, one of the most striking features of a strongly interacting EWSBS could be the appearance of resonances. For instance, for the MSM with $M_{H} \simeq 1 \mathrm{TeV}$, we expect a very broad scalar resonance around $1 \mathrm{TeV}$. In QCD-like models one expects a vector resonance (similar to the $\rho$ in pion physics) around $2 \mathrm{TeV}$.

However, the chiral formalism by itself is not able to reproduce resonances. Their very existence is closely related to the saturation of unitarity. But the chiral amplitudes do not even satisfy the elastic unitarity condition

$$
\operatorname{Im} t_{I J}(s)=\sigma(s)\left|t_{I J}(s)\right|^{2},
$$

where $\sigma(s)$ is the two-body phase space. Nevertheless, they satisfy it perturbatively

$$
\operatorname{Im} t_{I J}^{(1)}(s)=\sigma(s)\left|t_{I J}^{(0)}(s)\right|^{2}+O\left(s^{3}\right) .
$$

Notice that the violation of unitarity is very small only at low energies.

Therefore, in order to accommodate resonances we have to unitarize the chiral amplitudes. There are many math- ematical tricks to impose unitarity, which very often lead to different results. Obviously, that is the main criticism to unitarization. There is, however, a method that has been tested in ChPT and is able to reproduce the $\rho$ and $K^{*}$ resonances [10-12]. It is based on dispersion theory and apart from satisfying Eq. (5), it provides the correct unitarity cut on the complex $s$ plane, as well as poles in the second Riemann sheet.

\section{E. The inverse amplitude method}

Elastic amplitudes in the complex $s$ plane have a left and a right (or unitarity) cut. A dispersion relation is nothing but Cauchy's Theorem applied to these amplitudes. They are very useful since we can obtain the values of the amplitude in any point in terms of integrals of their imaginary parts over the cuts.

We have just seen that chiral amplitudes are not a good approximation at high energies on the elastic cut. Thus, they are not very well suited for a dispersive approach. The key point is to notice that we can calculate the imaginary part of the inverse amplitude exactly on the elastic cut. Indeed, using Eqs. (5) and (6),

$$
\operatorname{Im} \frac{1}{t_{I J}}=-\frac{\operatorname{Im} t_{I J}}{\left|t_{I J}\right|^{2}}=-\sigma .
$$

We can thus write a dispersion relation for $1 / t_{I J}$ whose integral over the elastic cut is exact. Nevertheless, the other analytical features are still approximate. In the Appendix, we give a detailed derivation and we comment on these approximations. Finally, it is possible to solve for $t_{I J}$ and we get

$$
t_{I J} \simeq \frac{t_{I J}^{(0) 2}}{t_{I J}^{(0)}-t_{I J}^{(1)}} .
$$

That is the IAM. Apart from its simplicity, it has several advantages.

(i) At low energies it reduces again to the very same chiral amplitudes in Eq. (3).

(ii) It satisfies elastic unitarity, Eq. (5), exactly.

(iii) The right cut is correctly reproduced and we get the appropriate analytic structure. In particular we get those poles in the second Riemann sheet which are near the unitarity cut [12].

(iv) It can be easily extended to higher orders $[11,12]$.

Of course it also has limitations. We comment on them thoroughly in the Appendix. However, they are mostly related to analytical structures (such as some poles or the left cut), which are far away from the energy range where we expect the resonances or unitarity effects. In the elastic region we expect the IAM to be a good approximation.

Indeed, the IAM has been applied both to pion elastic scattering and $\pi K$ scattering. The first example is very similar to the EWSBS, although there the GB are massive. In both cases it is possible to reproduce the lowest lying resonances: The $\rho(770)$ and the $K^{*}(892)$ respectively [10-12]. When only low-energy data is used, their masses lie about $15 \%$ off from the actual values. It is, however, possible to fit 

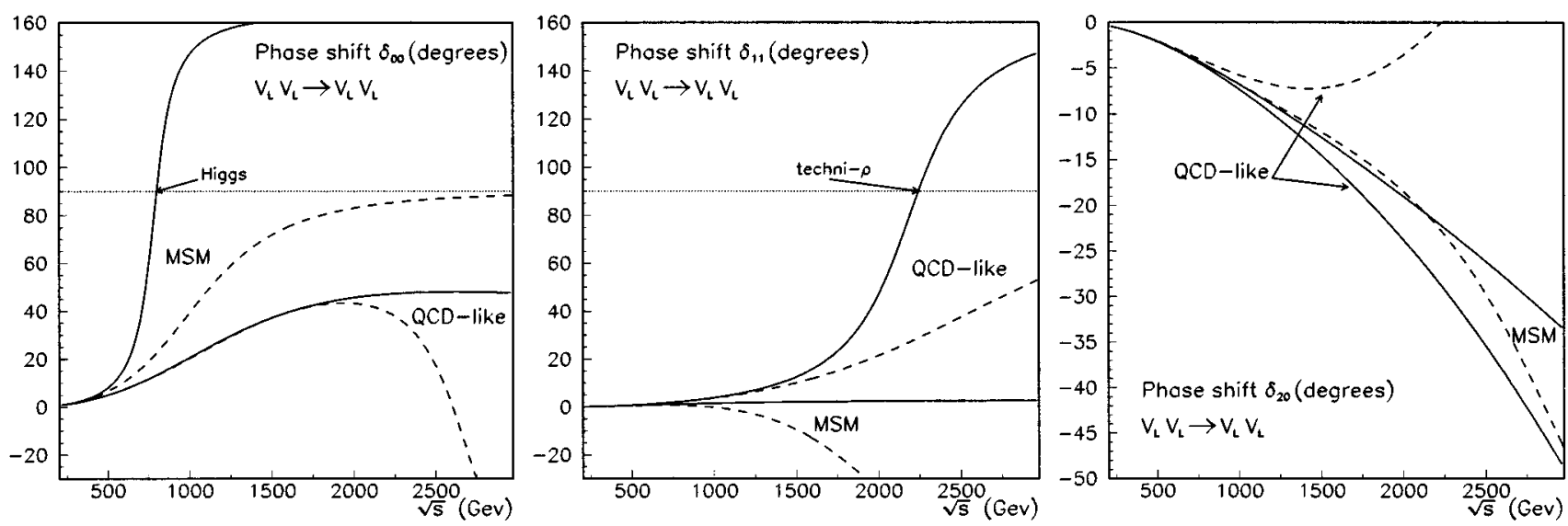

FIG. 1. Phase shifts in strong $V_{L} V_{L}$ scattering. The dashed lines are the plain chiral amplitudes and the continuous lines those using the IAM. They have been obtained both for a heavy-Higgs MSM and a QCD-like model using the parameters in Table I.

the masses and widths using high-energy data. Notice that this can be achieved without introducing any other field or parameter.

It is also important to remark that the IAM also improves considerably the nonresonant channels $[11,12]$. In fact, the $I=2$ channel in $\pi \pi$ and $I=3 / 2$ in $\pi K$ scattering do not present any low resonance. In spite of that, the results of the chiral amplitudes only match the data at low energies. The IAM results fit the data remarkably well up to much higher energies.

In addition the appearance of resonances is completely consistent with the QFT description. They are associated to poles in the second Riemann sheet, whose position is correctly related to the physical mass and width. The analytical structure of the IAM amplitudes is the correct one in the elastic region. We therefore consider that the chiral formalism, together with the IAM, is a reliable method to obtain, at least, a qualitative description of the resonance spectrum in strongly coupled systems.

\section{RESULTS}

\section{A. Reference models: Resonances and saturation}

The IAM in the chiral Lagrangian context was first applied to a MSM and a QCD-like model in [13]. There it was shown that it is possible to reproduce the expected reso- nances: a broad scalar resonance in the heavy Higgs MSM, and a technirho at about $2 \mathrm{TeV}$ in the QCD-like model. As an illustration, we show in Fig. 1 the phase shifts obtained when the IAM is applied to the chiral amplitudes.

They have been obtained using the parameters given in Table I, which have been actualized. Naively, the resonant masses can be obtained from the point where the corresponding phase shift crosses $90^{\circ}$. The width can be obtained assuming the typical Breit-Wigner for the $\rho$-like resonance and $M_{\rho} \simeq 2240 \mathrm{GeV}, \Gamma_{\rho} \simeq 620 \mathrm{GeV}$ shape. Their values are $M_{s} \simeq 800 \mathrm{GeV}, \Gamma_{s} \simeq 185 \mathrm{GeV}$ for the Higgs-like resonance. Notice that in this work we are also giving the results for the $I=2$ channel. It is related to like-sign pair production of gauge bosons, where the signal to background ratio seems very favorable, as it has been pointed out in $[17,20]$. In Fig. 1 it can be seen that the results using the IAM may vary significantly from those without unitarization. For instance, in the QCD-like case even the qualitative behavior is completely different. Comparing with QCD data, the correct behavior is the one given by the IAM $[11,12]$.

Finally, in Fig. 2, we show the position of the poles in the second Riemann sheet. Figure 2(a) is the pole that appears in the $(I, J)=(0,0)$ channel when using the MSM parameters of Table I. Figure 2(b) is the one that appears in the vector channel when using the QCD-like parameters. Notice that
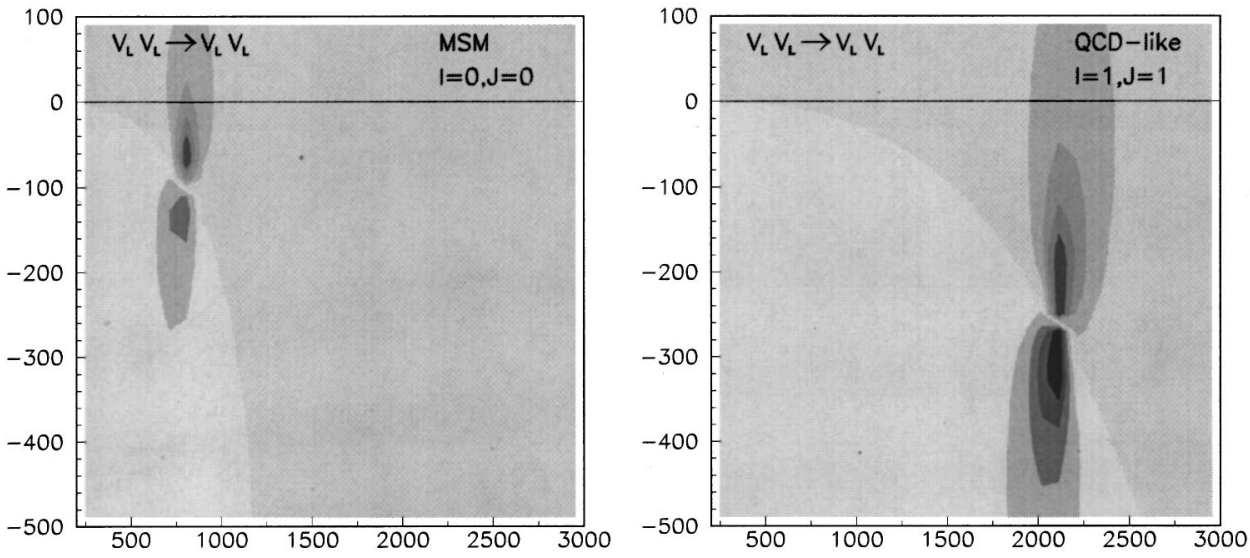

FIG. 2. Contour plots of the imaginary part of the $V_{L} V_{L}$ $\rightarrow V_{L} V_{L}$ chiral amplitudes. It has been extended continuously through the cut. Thus, above the real axis (straight line) is the first Riemann sheet, and below the second. (a) Pole of the scalar resonance in the $M_{H}=1 \mathrm{TeV}$ MSM. (b) Pole of the $\rho$-like resonance in the QCD-like channel. 

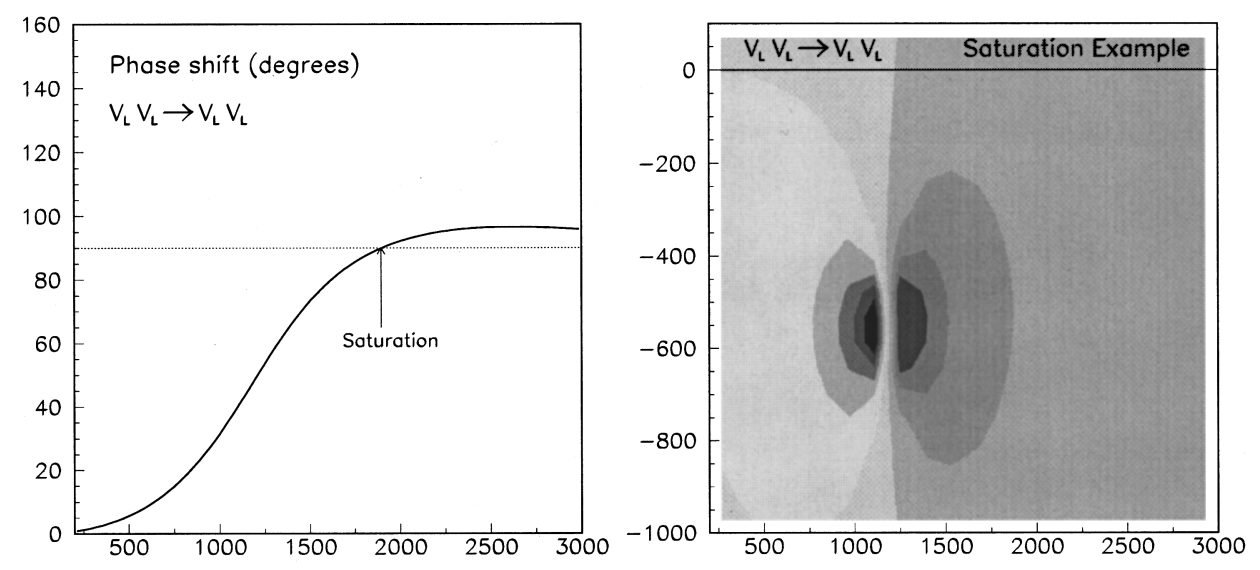

FIG. 3. On the left we show the phase shift of a channel displaying a "saturation" effect. For the same model we show on the right the contour plots of the imaginary part of the amplitudes. Notice the change of the scale with respect to Fig. 2. Observe that the pole is far away from the axis and has changed the orientation too.

the positions of the poles satisfy $\sqrt{s_{\text {pole }}} \sim M_{\text {res }}+i \Gamma_{\text {res }} / 2$.

\section{B. The scalar and vector channels}

\section{Saturation}

We have been paying special attention to resonances, but there are other interesting features. In particular, it could happen that the amplitude saturates unitarity although there is no clear resonant shape. At this point it is important to notice that the criterion of $\delta_{I J}$ crossing $90^{\circ}$ is only applicable to the cleanest cases. A resonance should be associated with a pole near the real axis which causes a steep raise in the phase shift. This pole reflects the existence of an almost bound state. When there is no other phase background this leads to our naive $90^{\circ}$ criterion. In such cases we can apply the usual Breit-Wigner description and relate, as above, the resonance physical constants with the pole position.

But it could well happen that there is a big phase shift background without a nearby pole. Then the phase shift can cross $90^{\circ}$ and saturate unitarity but we will not see the sudden increase in the phase shift. That we will call "saturation." As a matter of fact such big background phases are also produced by poles, but they are very far away from the real axis. Then it is either possible to say that there is no resonance or a very broad one. That is, for instance, the case of the $(I, J)=(0,0)$ channel in $\pi \pi$ scattering. That channel has a huge enhancement in the phase shift that grows very rapidly at small energies (see Fig. 1, which is a rescaled version). Such an enhancement has sometimes been interpreted as a resonance: the $\sigma$ particle. We will not address the $\sigma$ problem here. The only thing that is more or less clear is that such rapid enhancement should be produced by a pole [21] which is not very close to the real axis. Such a pole has been found using the IAM and ChPT in approximately the correct position [12].

The position of the poles in our amplitudes does obviously depend on the chiral parameters. Thus by varying $L_{1}$ and $L_{2}$ we can move the pole far away from the real axis and create such saturation effects. In Fig. 3 can be seen an example of that situation. Following the discussion above, the pole is much farther away from the real axis than those in Fig. 2. As a consequence, the Breit-Wigner relations between its position and the physical parameters of an hypothetical resonance, no longer hold. Notice also that the pole has changed its orientation.

\section{Resonances in parameter space}

We have seen that the IAM and the chiral formalism yields reasonable results in both reference models. Not only in terms of resonances but also in nonresonant channels. We have also shown how the different features are described accordingly to the requirements of analyticity and dispersion theory. Let us then explore the chiral parameter space in order to get a qualitative description of the possible EWSBS.

In Fig. 4 we show different contour plots in the $L_{1}, L_{2}$ plane. We display the $10^{-2}$ to $10^{-3}$ range, since generically we expect the parameters to be of that order.

The contour plots have been obtained from the calculation of the phase shifts in a $60 \times 60$ grid. Using these phase shifts, we have extracted two parameters: $M$, which is the energy at which $\delta_{I J}=90^{\circ}$ and

$$
\Gamma \simeq\left(M \frac{d \delta(s)}{d s}\right)^{-1}
$$

The interpretation of these parameters has to be made carefully. When $\Gamma \ll M$ they correspond to the mass and the width of a resonance in the Breit-Wigner approximation. Otherwise, the situation is similar to our previous "saturation" example and $M$ is just the point where the amplitudes saturate unitarity. In such a case, $\Gamma$ should not be interpreted as the width of a particle, although the saturation shape is broader for bigger $\Gamma$. In addition, $M$ and $\Gamma$ are not related to the pole position as in the Breit-Wigner formula. Remember from Fig. 3 that the pole not only moves away from the real axis, but it also changes its orientation.

We are showing three plots for the $(I, J)=(0,0)$ and $(1,1)$ channels separately. The contour plot on the left shows the values of $M$. That on the center is a contour plot of $\Gamma$. In order to clarify the meaning of these parameters, but also to get a qualitative picture of the many possible strong scenarios, we have added a third plot on the right. The dark gray area corresponds to "narrow" resonances. For illustrative purposes, we define narrow as $\Gamma<M / 4$. Roughly, this is what is usually understood by a resonance. Indeed, in QCD both the $\rho(770)$ and $K^{*}(892)$ satisfy this criterion. The light gray area stands at those $L_{1}, L_{2}$ values where we get a broad resonance. In this case, broad means $\Gamma>M / 4$ but even though the width is not very small, it is still possible to describe it with a pole and a Breit-Wigner description. Obviously, if we make the $\Gamma / M$ ratio even bigger the Breit- 

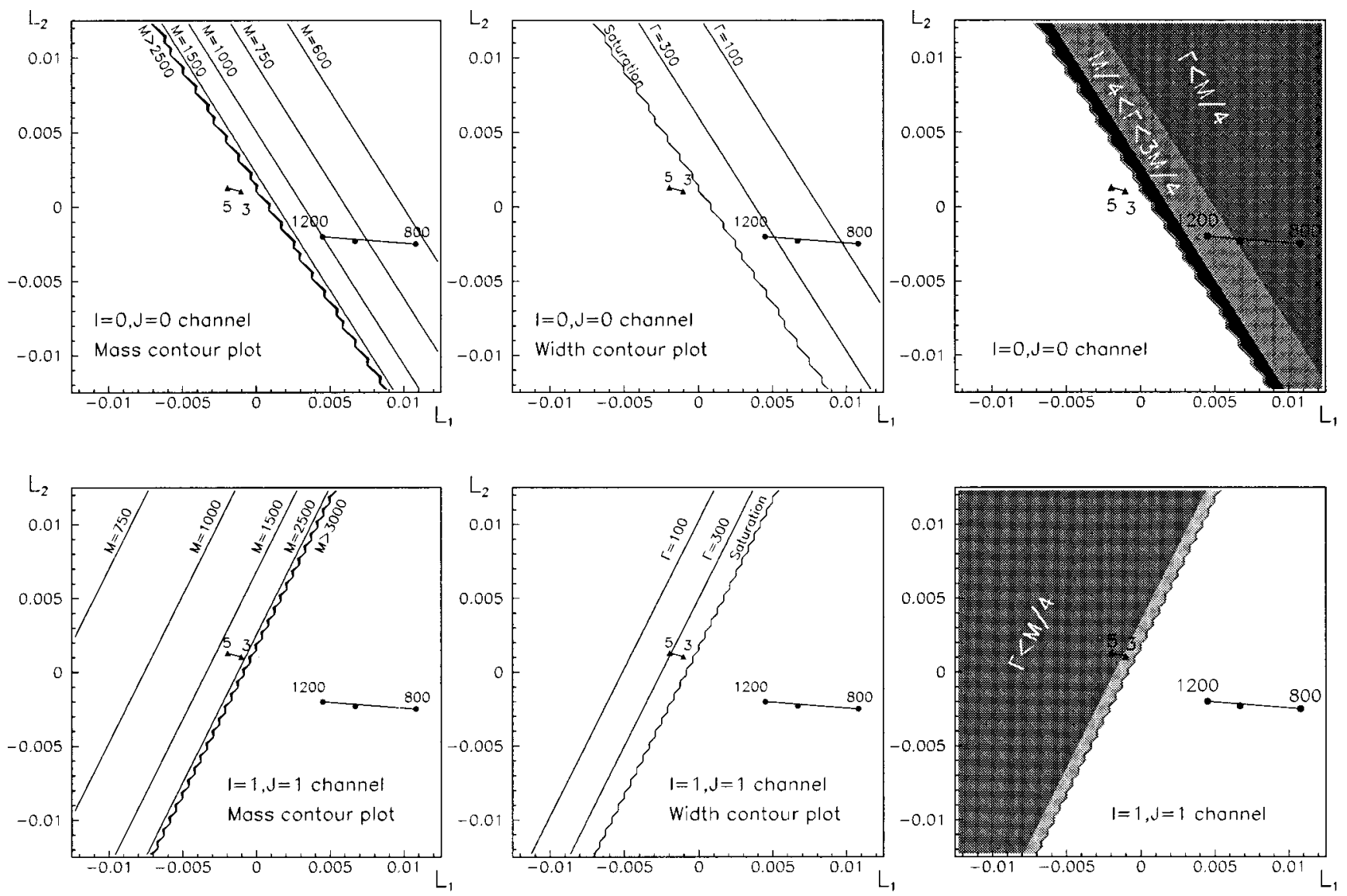

FIG. 4. Plots in the $L_{1}, L_{2}$ plane for the $(I, J)=(0,0)$ and $(1,1)$ channels. The plots on the left give contour levels of $M$. Those on the center give $\Gamma$. The dark grey areas stand for narrow $(\Gamma<4 M)$ resonances. The light grey areas for broad $(M / 4<\Gamma<3 M / 4)$ resonances and the black areas for saturation. White is no resonance or saturation below $3 \mathrm{TeV}$. The black circles stand at the values of $L_{i}$ that mimic a MSM with $M_{H}=800,1000$, and $1200 \mathrm{GeV}$. The black triangles represent QCD-like models with 3 or 5 colors.

Wigner description is no longer valid. That happens more or less at about $\Gamma>3 M / 4$ and at those points the black area starts, pointing the existence of a saturation effect.

\section{The $I=2$ channel}

We have already reviewed how the IAM in the chiral approach is able to reproduce the $I=0$ or $I=1$ resonances of our reference models. In the literature, there have also been proposed models with $I=2$ resonances (see [22] or [23], and references therein). However, they do not correspond to the kind of models that we are dealing with, since they always present light resonances or states. Indeed, in [22] two models were built with $I=2$ resonances, one of them with elementary and the other with composite doubly charged states. In both cases their masses are $M_{++} \leqslant 160 \mathrm{GeV}$ and there are also single charge states with $M_{+} \simeq 100 \mathrm{GeV}$.

Even more, the authors in [23] slightly modified the MSM including an $I=2$ resonance. Using a tree level unitarity, they found that the model does not make sense if its mass is bigger than $\sim 375 \mathrm{GeV}$. That bound becomes even smaller as the scalar Higgs-like resonance gets heavier. In the literature there are no models with an $I=2$ resonance and without light modes at the same time, that respect the custodial symmetry.

Within our approach, we find a similar result but for the general case. As soon as an $I=2$ resonant shape appears in the spectrum, the models do not make sense. Indeed, they present poles in the first Riemann sheet, within the IAM applicability region.

\section{Poles in the first sheet}

The $(I, J)=(2,0)$ phase shift is negative and that can give rise to several problems related to causality. In fact, saturation can also occur at $\delta_{20}=-90^{\circ}$. However, if we apply blindly Eq. (9), we get a negative value. Thus, even when $|\Gamma| \ll M$ we cannot say that there is a resonance, since its width would be negative. From the analytical point of view, that situation corresponds to a pole in the first Riemann sheet, which is forbidden.

As a matter of fact, the IAM yields poles in the first Riemann sheet of the $(I, J)=(2,0)$ amplitude. For instance, it is possible to find poles in $t_{20}$ in the first Riemann sheet at about $\sqrt{s_{\text {pole }}} \sim 3300+i 1750$ and $\sqrt{s_{\text {pole }}} \sim 4700+i 7000$ for the QCD-like and MSM parameters of Table I. However, in the chiral approach we are only allowed to use the IAM for energies $\sqrt{s} \lesssim 4 \pi v \sim 3 \mathrm{TeV}$. We should not worry about the IAM results outside that region, since it is not a good approximation there. These poles are well outside a circle of that radius in the complex plane and are not real predictions of the approach. In addition, when looking at pion physics, the description of $\delta_{20}$ is correct with the IAM and qualita- 
tively wrong (at high energies) with plain ChPT [11,12].

The problem is that the position of those poles depends on $L_{1}$ and $L_{2}$. In fact, it is possible to bring them close to the real axis and then the amplitudes do not have a physical meaning.

Let us now recall that the chiral Lagrangian does not meet all the requirements of a relativistic QFT. It respects Hermiticity, its amplitudes present a cut and an analytic structure, etc., but it is not renormalizable. It could well happen that, given a set of chiral parameters, there is no underlying theory consistent with all the QFT requirements. That could be enough to yield poles in the first Riemann sheet. If we were able to develop a method to detect those poles, we could rule out that parameter set as unphysical. In the Appendix we have shown that the IAM is able to reproduce these poles when they are present in the underlying theory.

The next step is to define how far these conflictive poles should be to accept the IAM results. Looking at the MSM and QCD-like examples, we notice that they are not a problem if they lie outside a $4 \pi v=3 \mathrm{TeV}$ circle in the complex plane. However, the IAM is a good approximation only near the real axis and thus the above criterion could be too strict. There is a much more intuitive criterion in order to exclude some values of $L_{1}$ and $L_{2}$.

\section{Wigner bound}

Indeed, there is a lower bound on the phase shift derivative due to Wigner [24]. Roughly it can be understood as follows. Phase shifts can be interpreted as the delay of the outcoming wave with respect to the incoming one. When it is negative, the outcoming signal is advanced. But that advance cannot be arbitrarily big. In the classical case, $d \delta / d k>-D$, where $D$ is the radius of the scatterer and $k$ the momentum of the incoming particle. The wave nature of particles does allow for a small violation of the previous equation. Near a resonance, it can be shown that $d \delta / d k>-(D+1 / 2 k)$ [24]. For a general potential the definition of $D$ is not so evident, but intuitively it has to be related to its effective size or range. Notice that this bound is valid for the elastic case.

Let us then translate the above arguments to our problem. First, in $V_{L} V_{L}$ scattering we are interested in the c.m. frame, where the momentum is $q^{2}=s / 4-m_{W}^{2}$. Second, we have been using the $\Gamma$ parameter instead of the slope. Using Eq. (9) our previous bound, in the c.m., reads

$$
\begin{aligned}
|\Gamma| & >\frac{2 m}{M} \frac{1}{D+m /\left(M^{2} \sqrt{1-4 m^{2} / M^{2}}\right)} \\
& \simeq \frac{2}{M /\left(8 \pi v^{2}\right)+1 /\left(M \sqrt{1-4 m^{2} / M^{2}}\right)}
\end{aligned}
$$

where in the last step we have used as $D$ the scattering length of the $t_{20}$ wave, which is the one we are interested in. It seems to be a reasonable estimate of the effective size of the potential. We will have to check that our results respect this condition. To start with, both reference models satisfy it. Let us now see what happens for other $L_{1}, L_{2}$ values.

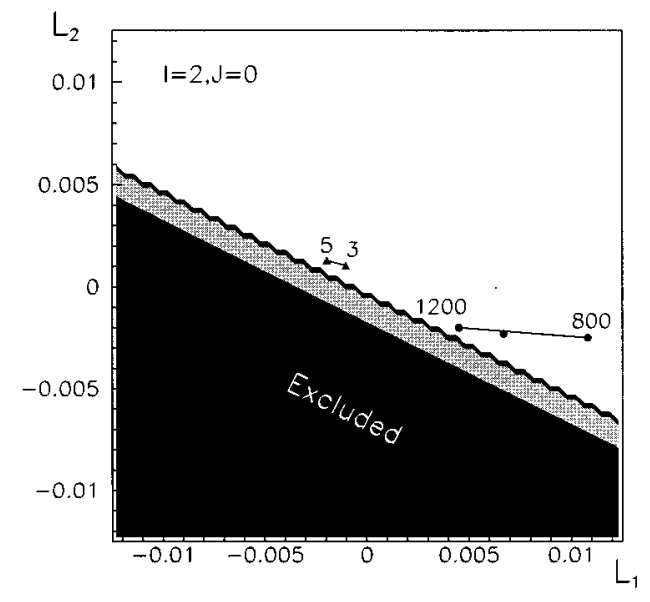

FIG. 5. Contour plot in the $L_{1}, L_{2}$ plane. The black area is excluded using the slope criterion and the IAM. The shaded area indicates a broad saturation shape in the cross section. In the white area there is no saturation below $3 \mathrm{TeV}$.

\section{The IAM results}

In Fig. 5 is shown the result of applying the bound in Eq. (10) to the IAM $t_{20}$ amplitude. The area in black represents the area excluded, whereas the white area is no saturation of unitarity. Notice that there is only a very narrow strip where the criterion is respected and saturation occurs. In this band, colored in grey, the saturation point $M$ is always reached above $M>2150 \mathrm{GeV}$, with $|\Gamma|>1050$. Surprisingly, the allowed $M$ and $\Gamma$ values are outside a $3-\mathrm{TeV}$ region. But that is again the first naive criterion of $\left|s_{\text {pole }}\right|>4 \pi v$. Thus, our allowed parameters yield amplitudes that satisfy both criteria at the same time. In the cross section these $M$ and $\Gamma$ parameters would give a very broad shape of a resonance (although it cannot be interpreted as a particle) or a saturation effect.

\section{DISCUSSION}

In the previous sections we have obtained the resonance spectrum for the general strong EWSBS. Let us now review the physical meaning of the results in the different $(I, J)$ channels.

$(\mathbf{0 , 0})$ channel. Concerning the MSM, we have already remarked that the IAM yields a Higgs-like resonance. As can be seen in Fig. 4, its mass is always smaller than $M_{H}$. As long as $M_{H}$ is the only relevant parameter, for a given resonance mass there is a fixed value of the width. With respect to QCD-like models, we do not get any resonance, but we get a considerable enhancement in this channel. This is the analogous of the $\sigma$ particle problem in QCD. There is also a pole very far from the real axis and it does not saturate unitarity.

In the general case, once we fix $M$ we get a unique $\Gamma$ too, since this channel only depends on the $8 L_{1}+5 L_{2}$ combination. That is not in conflict with existing models where the mass and the width of a scalar resonance can be adjusted [23] independently. In those models, there are resonances whose masses are $\sim 100 \mathrm{GeV}$. In this work we are only studying those models without low lying resonances. In addition, we have simplified the calculation to lowest order in $g$. When further corrections are included, other $L_{i}$ come into 
play and different values would change $\Gamma$. Nevertheless these effects are weaker and the variations should be relatively small. Let us also notice that we can get narrow resonances, broad resonances and that saturation occurs when $M \gtrsim 1500$ (and then we cannot strictly speak of a mass).

$(1,1)$ channel. Again there is only one $\Gamma$ for every $M$, since this channel only depends on $L_{2}-2 L_{1}$. In contrast with the previous channel, we can see in Fig. 4 that there are narrow resonances up to $M \$ 2500$. The values where we obtain a broad resonance are limited to a thin band, and we do not find what we have called "saturation" below $3 \mathrm{TeV}$.

The IAM yields a clear resonance in the QCD-like models. It is very narrow although not as much as the real $\rho$. That is due to the fact that in QCD the GB (the pions) are proportionally more massive than their analogous here (the $V_{L}$ ). It is also interesting to notice that vectorlike resonances become lighter when we assume more technicolors. As a consistency check, we do not get any resonance for the MSM.

$(\mathbf{2 , 0 )}$ channel. The interpretation of the results in this channel is more delicate. The IAM is only expected to work near the elastic cut. It has been tested in pion physics [12] and it yields the correct behavior in this channel. Nevertheless in ChPT it presents poles in the first Riemann sheet, although very far from the IAM applicability region. They cannot be considered predictions of the approach. However, the position of these poles depends on the chiral parameters, and it is indeed possible to get them very near the axis.

At this point we should remember that the chiral formalism is not renormalizable. It is not guaranteed that for every value of $L_{1}$ and $L_{2}$ there should be an underlying consistent theory. We have shown in the Appendix that in case these inconsistencies caused the appearance of a pole in the first sheet, and close to the unitarity cut, the IAM should be able to reproduce it properly. Consistently, when these poles are present we violate Wigner's bound on the phase shift slope. This bound is respected when we take the poles very far away.

We therefore consider the existence of those poles and the violation of the Wigner bound as a strong hint that the corresponding $L_{1}$ and $L_{2}$ are not allowed. In Fig. 5 we have shown the corresponding excluded region and those values where we get a saturation of unitarity, which always occurs at $M \gtrsim 2000$. In any case these parameters should never be mistaken for those of a resonance. Notice, once more, that the $\Gamma$ parameter is fixed for a given $M$. That is due to the fact that this channel only depends on $L_{1}+2 L_{2}$.

The most striking consequence of this result is that there cannot be narrow heavy $I=2$ resonances unless some of our initial assumptions are violated. Similar conclusions were found when trying to build models with such $I=2$ resonances $[22,23]$. It was not possible to make the $I=2$ resonances heavy unless the other particles in the spectrum become very light. Even in that case, the $I=2$ resonances were never bigger than $\sim 375 \mathrm{TeV}$.

\section{CONCLUSIONS}

In this work we have used the chiral Lagrangian approach to describe, with basically two parameters, the symmetrybreaking sector of the SM. Indeed, to any strong model respecting the custodial symmetry and without light reso-

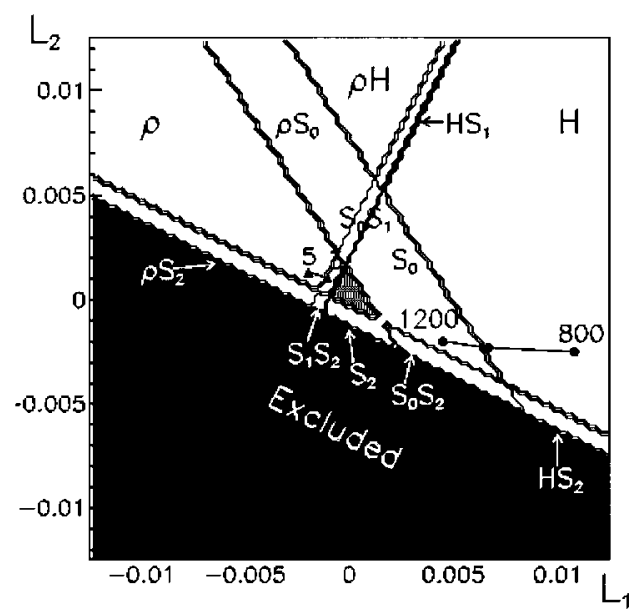

FIG. 6. Resonance spectrum of the strong EWSBS in the $L_{1}, L_{2}$ plane. The black area is excluded. On the white areas, we have represented broad resonances or saturation effects in the $I$ channel by $S_{I}$; Higgs-like narrow resonances by $\mathrm{H}$ and $\rho$-like narrow resonances by $\rho$. In the grey area there is no saturation of unitarity, nor resonances, below $3 \mathrm{TeV}$. The black dots represent the MSM with $M_{H}=800,1000,12000 \mathrm{GeV}$ and the triangles a QCD-like model with 3 or 5 technicolors.

nances, should correspond a value of this two parameters. However, it is not ensured that for any two parameters there should be an underlying consistent theory. By means of the inverse amplitude method, we have scanned this twodimensional parameter space in search for resonances or unitarity saturation effects.

We have reviewed how this approach is able to reproduce the expected behavior of popular models like the minimal SM or a QCD-like model. Within the expected parameter range, it is possible to find narrow resonances, broad resonances, or simply saturation of unitarity in both the $I=0$ or $I=1$ weak isospin channels. We have shown that the description of these resonances is consistent with the requirements of relativistic quantum field theory. Indeed, they are accompanied by poles in the second Riemann sheet whose position is correctly related to the resonance mass and width.

Concerning the $I=2$ channel, we have found that imposing elastic unitarity through the dispersive approach leads, for some values of the parameters, to poles in the first Riemann sheet. We consider that as a strong argument for excluding those values as unphysical. As a consequence, it does not seem possible to find heavy $I=2$ resonances in models respecting the above assumptions. That is in agreement with previous observations concerning specific models with $I=2$ resonances. Our result refers to the general strong scenario. Nevertheless, it seems still possible to have very broad shapes of unitarity saturation.

We have summarized the above results in Fig. 6. We have colored the excluded area in black. The white areas are labeled according to their unitarity features. There are two possible kinds of narrow resonances: a Higgs-like $(H)$ or a technirho $(\rho)$. By narrow we mean that the width is less than one fourth of the mass. We have denoted a broader saturation shape in the $I$ channel by $S_{I}$. Notice that, in contrast to the most popular models, it is possible to have two narrow resonances, a resonance in one channel and saturation in another, 
or saturation in two channels. Finally, the grey area correspond to those parameters that do not saturate unitarity below $3 \mathrm{TeV}$. For those models it is quite likely that the future colliders will not give even a hint on the nature of the electroweak symmetry-breaking sector.

\section{ACKNOWLEDGMENTS}

I would like to thank the Theory Group at Berkeley for their kind hospitality and the Jaime del Amo Foundation for financial support. I have also greatly appreciated the patience of M. Suzuki during his explanations and discussions on the unphysical poles. I am also indebted to R.S. Chivukula for his comments on models with $I=2$ resonances. This work has been partially supported by the Ministerio de Educación y Ciencia (Spain) (Grant No. CICYT AEN93-0776). Partial support by U.S. DOE under Contract No. DE-AC0376 SF00098 is gratefully acknowledged.

\section{APPENDIX: THE DERIVATION OF THE IAM}

In this appendix we will derive the IAM method using dispersion relations. Let us first remember that an elastic amplitude has a left and right (or elastic) cut and thus two Riemann sheets. A dispersion relation is nothing but Cauchy's theorem applied to one of these sheets. As a technical remark, let us notice that our amplitudes are $O\left(p^{4}\right) \sim O\left(s^{2}\right)$. Hence, we will have to divide by $s^{3}$ to ensure the vanishing of the closing integral contour at $\infty$. That is, elastic chiral amplitudes satisfy

$$
\begin{aligned}
t_{I J}(s)= & C_{0}+C_{1} s+C_{2} s^{2}+\frac{s^{3}}{\pi} \int_{\left(M_{\alpha}+M_{\beta}\right)^{2}}^{\infty} \frac{\operatorname{Im} t_{I J}\left(s^{\prime}\right) d s^{\prime}}{\left(s^{\prime}-s-i \epsilon\right)} \\
& +L C\left(t_{I J}\right) .
\end{aligned}
$$

The $C_{i}$ subtraction constants can be determined from the chiral approach.

Of course we only know how to calculate $t_{I J}^{(0)}$ and $t_{I J}^{(1)}$ which is just a crude approximation to the above relations:

$$
\begin{aligned}
t_{I J}^{(0)}= & a_{0}+a_{1} s, t_{I J}^{(1)}=b_{0}+b_{1} s+b_{2} s^{2} \\
& +\frac{s^{3}}{\pi} \int_{\left(M_{\alpha}+M_{\beta}\right)^{2}}^{\infty} \frac{\operatorname{Im} t_{I J}^{(1)}\left(s^{\prime}\right) d s^{\prime}}{\left.s^{\prime}-s-i \epsilon\right)} \\
& +L C\left(t_{I J}^{(1)}\right) .
\end{aligned}
$$

Our aim is to obtain a much better description of the right cut. That is because resonances are understood as poles in the second Riemann sheet, which is obtained continuously from that cut.

The relevant point is to realize that the inverse amplitude can be calculated exactly on the elastic cut. Indeed, using Eqs. (5) and (6) we find, on the right cut,

$$
\operatorname{Im} \frac{t_{I J}^{(0) 2}}{t_{I J}}=-t_{I J}^{(0) 2} \frac{\operatorname{Im} t_{I J}}{\left|t_{I J}\right|^{2}}=-t_{I J}^{(0) 2} \sigma=-\operatorname{Im} t_{I J}^{(1)}
$$

Notice that we have normalized the inverse amplitude with the real factor $t_{I J}^{(0) 2}$. Thus, apart from the poles, our function has the same analytic structure of $t_{I J}$. Observe that the poles of $t_{I J}$ are zeros of $G$ and vice versa. Thus we can write

$$
\begin{aligned}
\frac{t_{I J}^{(0) 2}}{t_{I J}} \simeq & a_{0}+a_{1} s-b_{0}-b_{1} s-b_{2} s^{2} \\
& -\frac{s^{3}}{\pi} \int_{\left(M_{\alpha}+M_{\beta}\right)^{2}}^{\infty} \frac{\operatorname{Im} t_{I J}^{(1)}\left(s^{\prime}\right) d s^{\prime}}{\left.s^{\prime}-s-i \epsilon\right)}-L C\left(t_{I J}^{(1)}\right)+P C(G) \\
\simeq & t_{I J}^{(0)}-t_{I J}^{(1)},
\end{aligned}
$$

where we have approximated $L C(G) \simeq L C\left(t_{I J}^{(1)}\right)$ and we have neglected $P C(G)$. That is

$$
t_{I J} \simeq \frac{t_{I J}^{(0) 2}}{t_{I J}^{(0)}-t_{I J}^{(1)}}
$$

which is the IAM method. In the text we have already commented its advantages, but there are also some limitations.

(i) We have only used elastic unitarity, and that limits the validity at high energies where the first two-body inelastic threshold appears [12].

(ii) We have also neglected the pole contributions of $G$ and thus we are not able to describe Adler zeros below threshold.

(iii) Finally, we have approximated the left cut of the inverse function by that of $t^{(1)}$. Hence we violate crossing symmetry. In addition we only reproduce the leading but not the subleading logarithms.

Notice, however, that the expansion of the IAM at low energies is again the chiral expansion $t_{I J} \sim t_{I J}^{(0)}+t_{I J}^{(1)}$ so that the error in this approximation is $O\left(s^{3}\right)$. At higher energies, the contribution from the left cut and poles below threshold become less relevant, due to the $\left(s^{\prime}-s\right)$ factor in the denominator. Their effect will be to change slightly the position of the resonance. In previous applications to ChPT it has been found that this shift is usually smaller than $15 \%$ [12]. As long as we are only interested in a qualitative description of resonances, they will be neglected. Very recently, however, an improved version of the IAM has been proposed [25], although it does not yield such a simple formula. That is why we will not use it here.

Finally, let us remark that we have only needed the dispersion relation for the inverse amplitude as well as those for the approximated amplitudes, which do not have poles. Even if the theory is pathological and presents poles in the first sheet, the IAM derivation is still valid. These poles in the amplitude become zeros of the inverse amplitude and they do not change the analytic structure. We can thus use the very same expression of the IAM in Eq. (8) to detect poles in the first Riemman sheet. However, we still have to remember that the approximations we have done limit the validity of the method to a region close to the elastic cut. Any feature, including poles, outside that region do not deserve any consideration. 
[1] M.S. Chanowitz, M. Golden, and H. Georgi, Phys. Rev. D 36, 1490 (1987).

[2] A. Dobado and M.J. Herrero, Phys. Lett. B 228, 495 (1989); 233, 505 (1989); J. Donoghue and C. Ramirez, ibid. 234, 361 (1990)

[3] S. Weinberg, Physica A 96, 327 (1979); J. Gasser and H. Leutwyler, Ann. Phys. (N.Y.) 158, 142 (1984); Nucl. Phys. B250, 465 (1985); B250, 517 (1985).

[4] T. Appelquist and C. Bernard, Phys. Rev. D 22, 200 (1980); A.C. Longhitano, Nucl. Phys. B188, 118 (1981).

[5] M.J. Herrero and E. Ruiz Morales, Nucl. Phys. B418, 431 (1994); B437, 319 (1995).

[6] See also, D. Espriu and J. Matias, Phys. Lett. B 341, 332 (1995); S. Dittmaier and C. Grosse-Knetter, Phys. Rev. D 52, 7276 (1995).

[7] T. Appelquist and G.-H. Wu, Phys. Rev. D 48, 3235 (1993).

[8] CMS Technical Proposal No. CERN/LHC94-38.LHCC/P1, 1994 (unpublished); A. Dobado, M.J. Herrero, J.R. Peláez, E. Ruiz Morales, and M.T. Urdiales, Phys. Lett. B 352, 400 (1995); A. Dobado and M.T. Urdiales, Z. Phys. C 71, 659 (1996).

[9] Tran N. Truong, Phys. Rev. Lett. 61, 2526 (1988); 67, 2260 (1991).

[10] A. Dobado, M.J. Herrero and T.N. Truong, Phys. Lett. B 235, 134 (1990).

[11] A. Dobado and J.R. Peláez, Phys. Rev. D 47, 4883 (1993).
[12] A. Dobado and J.R. Peláez, Report No. hep-ph/9604416 (unpublished).

[13] A. Dobado, M.J. Herrero, T.N. Truong, Z. Phys. C 50, 129 (1990); A. Dobado, M.J. Herrero, and J. Terrón, ibid. 50, 205 (1991); ibid. 50, 465 (1991).

[14] P. Sikivie et al., Nucl. Phys. B173, 189 (1980).

[15] A. Dobado and J.R. Peláez, Nucl. Phys. B425, 110 (1994).

[16] J.M. Cornwall, D.N. Levin, and G. Tiktopoulos, Phys. Rev. D 10, 1145 (1974); C.E. Vayonakis, Lett. Nuovo Cimento 17, 383 (1976); B.W. Lee, C. Quigg, and H. Thacker, Phys. Rev. D 16, 1519 (1977).

[17] M.S. Chanowitz and M.K. Gaillard, Nucl. Phys. B261, 379 (1985).

[18] Y.P. Yao and C.P. Yuan, Phys. Rev. D 38, 2237 (1988); J. Bagger and C. Schmidt, ibid. 41, 264 (1990); H. Veltman, ibid. 41, 2294 (1990); H.J. He, Y.P. Kuang, and X. Li, Phys. Rev. Lett. 69, 2619 (1992).

[19] H.J. He, Y.P. Kuang, and X. Li, Phys. Lett. B 329, 278 (1994).

[20] M. Berger and M.S. Chanowitz, Phys. Lett. B 236, 509 (1991).

[21] B.S. Zou and D.V. Bugg, Phys. Rev. D 48, 3948 (1993).

[22] H. Georgi and M. Machacek, Nucl. Phys. B262, 463 (1985).

[23] R.S. Chivukula, M.J. Dugan, and M. Golden, Phys. Lett. B 336, 62 (1994).

[24] E.P. Wigner, Phys. Rev. 98, 145 (1955); R.G. Newton, Scattering Theory of Waves and Particles (McGraw-Hill, New York, 1966).

[25] M. Boglione and M. R. Pennington, Report No. hep-ph/9607266 (unpublished). 